\title{
A SIMPLE PROOF OF THE CLASSIFICATION OF NORMAL TOEPLITZ MATRICES*
}

\author{
AKIO ARIMOTO ${ }^{\dagger}$
}

\begin{abstract}
An easy proof to show that every complex normal Toeplitz matrix is classified as either of type I or of type II is given. Instead of difference equations on elements in the matrix used in past studies, polynomial equations with coefficients of elements are used. In a similar fashion, it is shown that a real normal Toeplitz matrix must be one of four types: symmetric, skew-symmetric, circulant, or skew-circulant. Here trigonometric polynomials in the complex case and algebraic polynomials in the real case are used.
\end{abstract}

Key words. Normal matrices, Toeplitz matrices.

AMS subject classifications. 15A57, 47B15, 47B35

1. Introduction: Normal Toeplitz Matrices. Ikramov [3] first showed that a normal Toeplitz matrix over real field must be one of four types: symmetric, skewsymmetric (up to the principal diagonal), circulant, or skew-circulant. Later on, the complex version of the problem was solved by Ikramov et al. [6] and Gel'fgat [2] . The history of the theory was described in [5]. The complex case was also proved by Farenick, Krupnik, Krupnik and Lee [1], and Ito [2], independently of [6]. We give here another proof which, we believe, is the simplest one. The complex case and the real case will be proved in a similar fashion, although trigonometric polynomials will be used in the complex case and algebraic polynomials will be used in the real case. Now denote Toeplitz matrix of order $N+1$ by $T_{N}$ :

$$
T_{N}=\left[\begin{array}{ccccc}
a_{0} & a_{-1} & a_{-2} & \cdots & a_{-N} \\
a_{1} & a_{0} & a_{-1} & \cdots & a_{-(N-1)} \\
\cdots & \cdots & \cdots & \cdots & \\
a_{N-1} & a_{N-2} & a_{N-3} & \cdots & a_{-1} \\
a_{N} & a_{N-1} & a_{N-2} & \cdots & a_{0}
\end{array}\right]
$$

The matrix $T_{N}$ is called normal Toeplitz if $T_{N} T_{N}^{*}-T_{N}^{*} T_{N}=0$, where $T_{N}^{*}$ is the transposed conjugate of $T_{N}$. We can simply state classifications of normal Toeplitz matrices in the following way. A normal Toeplitz matrix $T_{N}$ is of type $I$ if and only if for some $\alpha_{0}$, with $\left|\alpha_{0}\right|=1$ and,

$$
\left[\begin{array}{llll}
a_{-1} & a_{-2} & \cdots & a_{-N}
\end{array}\right]=\alpha_{0}\left[\begin{array}{llll}
\bar{a}_{1} & \bar{a}_{2} & \cdots & \bar{a}_{N}
\end{array}\right]
$$

whereas $T_{N}$ is of type II if and only if for some $\beta_{0}$, with $\left|\beta_{0}\right|=1$,

$$
\left[\begin{array}{llll}
a_{-1} & a_{-2} & \cdots & a_{-N}
\end{array}\right]=\beta_{0}\left[\begin{array}{llll}
a_{N} & a_{N-1} & \cdots & a_{1}
\end{array}\right]
$$

${ }^{*}$ Received by the editors on 1 March 2002. Final manuscript accepted on 2 May 2002. Handling Editor: Harm Bart.

${ }^{\dagger}$ Department of Electronics and Computer Engineering, Musashi Institute of Technology, Tamazutsumi 1-28-1, Setagaya-ku, Tokyo 158, Japan (arimoto@iname.com). 
Surely the complex case contains the real case, but when all the $a_{k}^{\prime} s$ are real valued, it will be found that $\alpha_{0}$ and $\beta_{0}$ must be either +1 or -1 , hence we may have the further concrete classification of four types: A normal Toeplitz matrix $T_{N}$ is symmetric if and only if

$$
\left[\begin{array}{llll}
a_{-1} & a_{-2} & \cdots & a_{-N}
\end{array}\right]=\left[\begin{array}{llll}
a_{1} & a_{2} & \cdots & a_{N}
\end{array}\right]
$$

$T_{N}$ is skew-symmetric if and only if

$$
\left[\begin{array}{llll}
a_{-1} & a_{-2} & \cdots & a_{-N}
\end{array}\right]=-\left[\begin{array}{llll}
a_{1} & a_{2} & \cdots & a_{N}
\end{array}\right],
$$

$T_{N}$ is circulant if and only if

$$
\left[\begin{array}{llll}
a_{-1} & a_{-2} & \cdots & a_{-N}
\end{array}\right]=\left[\begin{array}{llll}
a_{N} & a_{N-1} & \cdots & a_{1}
\end{array}\right]
$$

$T_{N}$ is skew-circulant if and only if

$$
\left[\begin{array}{llll}
a_{-1} & a_{-2} & \cdots & a_{-N}
\end{array}\right]=-\left[\begin{array}{llll}
a_{N} & a_{N-1} & \cdots & a_{1}
\end{array}\right] .
$$

Throughout this paper, we assume $a_{0}$ to be zero without loss of generality since $a_{0}$ does not play any role in the above classifications.

2. Proof of Theorem in the Complex Case. Now we define trigonometric polynomials $s(x)$ and $t(x)$ with coefficients $a_{1}, a_{2}, \cdots, a_{N}$ and $a_{-1}, a_{-2}, \cdots, a_{-N}$, respectively:

$$
\begin{aligned}
& s(x)=a_{1} e^{i x}+a_{2} e^{2 i x}+\cdots+a_{N} e^{N i x} \\
& t(x)=a_{-1} e^{-i x}+a_{-2} e^{-2 i x}+\cdots+a_{-N} e^{-N i x} .
\end{aligned}
$$

Using these trigonometric polynomials, we can restate conditions (1) and (2) in the previous section as

(1') type I: $t(x)=\alpha_{0} \overline{s(x)}$, with $\left|\alpha_{0}\right|=1$,

(2') type II: $t(x)=\beta_{0} s(x) e^{-i(N+1) x}$, with $\left|\beta_{0}\right|=1$.

Theorem 2.1. Every normal Toeplitz matrix is either of type I or of type II.

Proof. Our proof is based on the expression in [7, p. 998] or [1, p. 1038]. Necessary and sufficient conditions for $T_{N}$ to be normal in terms of $a_{n}$ are that

$$
a_{m} \bar{a}_{n}-\bar{a}_{-m} a_{-n}+\bar{a}_{N+1-m} a_{N+1-n}-a_{-(N+1-m)} \bar{a}_{-(N+1-n)}=0,
$$

$(1 \leq n, m \leq N)$.

We now rewrite (7) by using polynomials $s(x)$ and $t(x)$. Multiply both sides of (7) by $e^{i m x} e^{-i n y}$ and sum these new expressions over all $m$ and $n$ to obtain

$$
s(x) \overline{s(y)}-\overline{t(x)} t(y)+\overline{s(x)} s(y) e^{i(N+1)(x-y)}-t(x) \overline{t(y)} e^{i(N+1)(x-y)}=0 .
$$

It easily seen that (8) is equivalent to (7), and that (1') or (2') implies (8). Hence both (1') and (2') are sufficient conditions for $T_{N}$ to be normal. Thus it only remains to show the necessity of the condition. When we take $x=y$ in (8), we have

$$
s(x) \overline{s(x)}-t(x) \overline{t(x)}=0, \text { or }|s(x)|=|t(x)|
$$


for each $x$. Hence except for trivial case $t(x) \equiv 0$, we can find an $x_{0}$ such that $t\left(x_{0}\right) \neq 0$ and there exist $\alpha$ and $\beta$ such that $s\left(x_{0}\right)=\alpha t\left(x_{0}\right)$ and $t\left(x_{0}\right)=\beta \overline{t\left(x_{0}\right)}$, with $|\alpha|=|\beta|=1$. By setting $y=x_{0}$ and then by dividing through by $\overline{t\left(x_{0}\right)}$, equation (8) becomes

$$
s(x) \bar{\alpha}-\overline{t(x)} \beta+\overline{s(x)} \alpha \beta e^{i(N+1)\left(x-x_{0}\right)}-t(x) e^{i(N+1)\left(x-x_{0}\right)}=0,
$$

from which we have

$$
\overline{t(x)}=s(x) \overline{\alpha \beta}+\overline{s(x)} \alpha e^{i(N+1)\left(x-x_{0}\right)}-t(x) \bar{\beta} e^{i(N+1)\left(x-x_{0}\right)} .
$$

Substituting the right hand side of (10) into $\overline{t(x)}$ of (9), we have

$$
\left\{s(x)-t(x) \alpha e^{i(N+1)\left(x-x_{0}\right)}\right\}\{\overline{s(x)}-t(x) \overline{\alpha \beta}\}=0,
$$

which implies our required results if we take $\alpha_{0}=\alpha \beta$ and $\beta_{0}=\bar{\alpha} e^{i(N+1) x_{0}}$. We should notice here that (11) is the product of polynomials. If one of the polynomials in (11) is nonzero at $x=x_{0}$, then by continuity the polynomial is nonzero in an open neighborhood $\mathcal{U}$ of $x_{0}$, which implies that the other polynomial in the product (11) is identically zero on $\mathcal{U}$. Because the only polynomial with a continuum of roots is the zero polynomial, equation (11) implies equation (1') or (2'). Hence type I or type II must occur.

\section{Proof of Theorem in the Real Case.}

THEOREM 3.1. Every real normal Toeplitz matrix is either symmetric, skewsymmetric, circulant or skew-circulant.

Proof. We define algebraic polynomials $p(x)$ and $q(x)$ such that

$$
\begin{aligned}
& p(x)=a_{1} x+a_{2} x^{2}+\cdots+a_{N} x^{N} \\
& q(x)=a_{-1} x+a_{-2} x^{2}+\cdots+a_{-N} x^{N} .
\end{aligned}
$$

Also in this real case we can apply (7) in the previous section as necessary and sufficient conditions for $T_{N}$ to be normal in terms of real valued $a_{n}$ :

$$
a_{m} a_{n}-a_{-m} a_{-n}+a_{N+1-m} a_{N+1-n}-a_{-(N+1-m)} a_{-(N+1-n)}=0(1 \leq n, m \leq N)
$$

Now we introduce the reciprocal polynomials to $p(x)$ and $q(x)$

$$
\tilde{p}(x)=a_{N} x+a_{N-1} x^{2}+\cdots+a_{1} x^{N}
$$

and

$$
\tilde{q}(x)=a_{-N} x+a_{-N+1} x^{2}+\cdots+a_{-1} x^{N},
$$

respectively. Multiply both sides of equation (12) by $x^{m} y^{n}$ and then sum the resulting expressions over all $m$ and $n$ to obtain the following analog of (8):

$$
p(x) p(y)-q(x) q(y)+\tilde{p}(x) \tilde{p}(y)-\tilde{q}(x) \tilde{q}(y)=0 .
$$


Noticing the relation $\tilde{q}(x)=x^{N+1} q(1 / x)$ and $\tilde{p}(x)=x^{N+1} p(1 / x)$, and letting $y=$ $1 / x$ in (13), we have $p(x) \tilde{p}(x)=q(x) \tilde{q}(x)$. By setting $x=y$ and then multiplying both sides of (13) by $p^{2}(x)$, we have from the relation $p^{2}(x) \tilde{p}^{2}(x)=q^{2}(x) \tilde{q}^{2}(x)$,

$$
p^{4}(x)-p^{2}(x) q^{2}(x)+q^{2}(x) \tilde{q}^{2}(x)-p^{2}(x) \tilde{q}^{2}(x)=0,
$$

which can be factored as the following way:

$$
\left(p^{2}(x)-q^{2}(x)\right)\left(p^{2}(x)-\tilde{q}^{2}(x)\right)=0 .
$$

¿From this formula, we obtain four types, symmetric $p(x)=q(x)$, skew-symmetric $p(x)=-q(x)$, circulant $p(x)=\tilde{q}(x)$ and skew-circulant $p(x)=-\tilde{q}(x)$, which are equivalent to $(3),(4),(5)$, and (6), respectively.

\section{REFERENCES}

[1] D.R. Farenick, M. Krupnik, N. Krupnik, and W.Y. Lee. Normal Toeplitz matrices. SIAM J. Matrix Anal. Appl., 17:1037-1043, 1996.

[2] V.I .Gel'fgat. A normality criterion for Toeplitz matrices. Zh. Vychisl. Mat. Math. Fiz., 35:14281432, 1995 (in Russian). Comput. Math. Math. Phys., 35:1147-1150, 1995.

[3] Kh.D. Ikramov. Describing normal Toeplitz matrices. Zh. Vychisl. Mat. Mat. Fiz., 34:473-479, 1994 (in Russian). Comput. Math. Math. Phys., 34:399-404, 1994.

[4] Kh.D. Ikramov. Classification of normal Toeplitz matrices with real entries. Mat. Zametki, 57:1428-1432, 1995 (in Russian). Math. Notes, 57:463-469, 1995

[5] Kh.D. Ikramov. Comments on Normal Toeplitz Matrices by Farenick et al. SIAM J. Matrix Anal. Appl., 18:218, 1997.

[6] Kh.D. Ikramov and V.N. Chugunov. Normality condition for a complex Toeplitz matrix. $Z h$. Vychisl. Mat. Mat. Fiz., 36:3-10, 1996 (in Russian). Comput. Math. Math. Phys., 36:131$137,1996$.

[7] T. Ito. Every normal Toeplitz matrix is either of type I or of type II. SIAM J. Matrix Anal. Appl., 17:998-1106, 1996. 